\title{
Analysis and modeling of spatial characteristics in urban microscenario of heterogeneous network
}

\author{
Jianhua Zhang ${ }^{1 *}$, Nan Sheng ${ }^{1}$, Fenghua Zhang ${ }^{1}$, Lei Tian ${ }^{1}$, Guangyi Liư ${ }^{2}$, Weihui Dong ${ }^{2}$, Ping Zhang ${ }^{1}$ and \\ Chia-Chin Chong ${ }^{3}$
}

\begin{abstract}
Heterogeneous network (HetNet) is a typical deployment scenario for the IMT-Advanced system whereby the macro enhanced node B (eNB) provides the wide coverage while the lower power nodes such as micro, pico, femto, and relay nodes extend the coverage/capacity for coverage hole or hotspot. This literature addresses the spatial propagation modeling for urban micro (UMi) scenario of HetNet. Due to users distributed in canyon streets, the multipath with high power is not always coming from the line-of-sight (LoS) direction in UMi scenario. Moreover, considering the impact of the directional antenna pattern, the current IMT-Advanced UMi channel model may lead to inaccurate interference modeling. To verify this, multiple-input multiple-output (MIMO) field channel measurement is conducted in downtown Beijing for typical UMi. Based on the measurement data analysis, the multipath's angular offset from the LoS direction is clearly observed. In order to capture such spatial characteristic into the existing IMT-Advanced UMi channel model, the angular offset models are proposed for both LoS and non-LoS (NLOS) cases. Finally, the interference and capacity simulation prove that it is necessary to capture the angle offset model into the MIMO channel model in UMi scenario.
\end{abstract}

Keywords: IMT-Advanced, multiple-input multiple-output (MIMO), channel model, interference, HetNet

\section{Introduction}

With the expansion of the mobile data market, the mobile operators have more and more pressure to expand the cellular capacity by cell splitting or carrier aggregation [1]. In order to make full use of the expensive spectrum, the cellular technology is required to improve the spectrum efficiency as much as possible. As reported in [2], the mobile data market will increase more than 50 times from 2010 to 2015 . In order to meet the requirements of the future data market, 3rd Generation Partnership Project (3GPP) has started the research and standardization of the next generation cellular network technology, which is called as LTEAdvanced [3].

The cellular system is usually planned as hierarchical coverage. The macro enhanced node B (eNB) with high transmit power and high antenna height is deployed to

\footnotetext{
* Correspondence: jhzhang@bupt.edu.cn

${ }^{1}$ Key Laboratory of Universal Wireless Communications, Ministry of Education, Beijing University of Posts and Telecommunications, P.O. Box 92, Beijing 100876, China

Full list of author information is available at the end of the article
}

provide wide coverage as the basic layer, whereas some low power nodes such as micro, pico, femto, and relay [4] nodes are deployed for the coverage/capacity expansion as the secondary layer. In order to alleviate the complexity of the network planning and optimization in hierarchical cellular deployment like Global System for Mobile (GSM) and Universal Mobile Telecommunication System, the macro eNB and micro/pico nodes are allocated with different carrier frequencies, and thus the interference between different coverage layers can be ignored.

According to the prediction from International Telecommunication Union-Radio communication sector (ITU-R) [5], the required spectrum for IMT-Advanced is above $1 \mathrm{GHz}$, while the spectrum allocated for the IMT-Advanced by ITU-R is less than $500 \mathrm{MHz}$ now. In order to fill the spectrum gap between the required and the available, more aggressive spectrum usage strategies have to be considered for IMT-Advanced. In 3GPP, the hierarchical network with the same spectrum allocated for both basic and secondary layers is defined as heterogeneous network (HetNet) [6]. Compared to the 
homogeneous interference among the macro eNBs, the heterogeneous interference between macro eNB and lower power nodes becomes more serious. In order to deal with the serious inter-cell interference, the enhanced inter-cell interference coordination (eICIC) [6] and heterogeneous coordinated multiple point transmission and reception (CoMP) $[7,8]$ are proposed in 3GPP.

To facilitate the corresponding performance evaluation for HetNet, 3GPP has defined the evaluation methodology for HetNet and eICIC [6]. However, only the path loss and shadow fading are explicitly defined based on the existing models, such as the IMT-Advanced model [9] and ITU-R M.1225 [10], whereas the fast fading is not defined explicitly. For the performance evaluation of eICIC in time domain, the path loss and shadowing may be sufficient; however, for the IICIC and CoMP in the spatial and frequency domains, the fast fading is necessary to show a reliable performance. Therefore, both the fast fading and slow fading of the MIMO channel should be captured in performance evaluation methodology of HetNet.

As shown in Figure 1, the eNB sites in typical urban scenarios are usually surrounded by high-rise buildings. For the outdoor users distributed in the streets outside of the building, the multipaths with high power are not always coming from the line-of-sight (LoS) direction due to the special street environment in urban micro (UMi) scenario. However, in the current geometry-based spatial channel model (GBSM), e. g., IMT-Advanced UMi channel model [9], the high power multipath always focuses around the LoS direction for user equipment (UE). Therefore, the conflict between the characteristics of the typical UMi scenario and its corresponding GBSM model may happen, which lead to inconsistency between the real propagation characteristics and the corresponding spatial channel modeling.

In this literature, the spatial models of the typical UMi scenario of HetNet are addressed. Regarding the existing IMT-Advanced UMi channel model, due to the impact of the directional antenna pattern of eNB transmitter and the phenomenon as described above, the intra-site interference from the neighboring sectors of the same micro eNB may be underestimated and thus, leads to overestimation of the single point MIMO system performance. Dedicated MIMO field channel measurements were conducted in downtown Beijing for typical UMi scenario. The angular offset of the multipath is observed from the measurement results, and a modified MIMO channel model is proposed to capture such spatial characteristics into the UMi channel model, where a random angular offset is captured into the fast fading.

To verify the influence of the proposed model, the theoretical channel capacity based on the measured data is analyzed, and system level simulations of Time Division LTE-Advanced (TD-LTE-Advanced) system [11] are performed. The numerical results show that the intra-site interference has been underestimated by the original IMT-Advanced UMi model, while the proposed model provide better CoMP gain due to taking into account the impact of the directional antenna pattern and the angular offset on the multipath.

The rest of the article is organized as follows. The limitations of the existing channel models are discussed in Section 2. The field channel measurement is described in Section 3. The proposed channel model for UMi is presented in Section 4. The theoretical analysis and system level simulation results are given in Sections 5 and 6, respectively. Finally, the conclusions are drawn in Section 7.

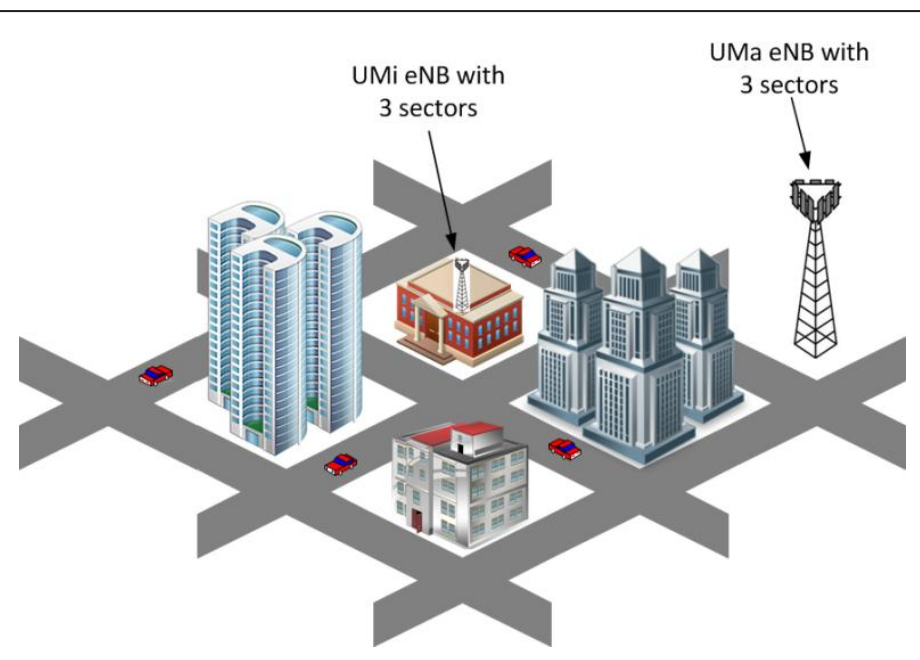

Figure 1 HetNet deployment of macro/micro in typical urban environment. 


\section{Limitations of existing channel model}

Wireless channel consists of many propagation paths, which diffuse in the spatial domain at both the transmitter and the receiver. The performance of MIMO system is greatly affected by the extent of the angular dispersion of angle of departure (AoD) and angle of arrival (AoA), which is described by the angular spread (AS) in the existing channel models. Moreover, due to the application of sectorized antennas, the spatial characteristics of the channel between the eNB and a certain UE may be influenced by the antenna pattern of the eNB.

As illustrated in Figure 2, the eNB has three sectors, i. e., sectors A, B, and C. UE is served by sector A. Without loss of generality, we take the downlink interference from sector B to the UE for example. The intra-site interference could arrive at the UE either straightly from the backside of the antenna (the green line in Figure 2) or from the reflection by remote scatterers (the red line in Figure 2). In existing GBSM, e.g., IMT-Advanced channel model [9], almost all the intra-site interference from sector B is supposed to come from the backside of sector $B$ antenna and has experienced extra $25 \mathrm{~dB}$ attenuation because of the front to back ratio of the antenna [9].

However, it might not be the case in UMi scenario, especially in the downtown of dense urban like Beijing. The canyon-like streets environment in such scenario may lead to peculiar spatial characteristics of the

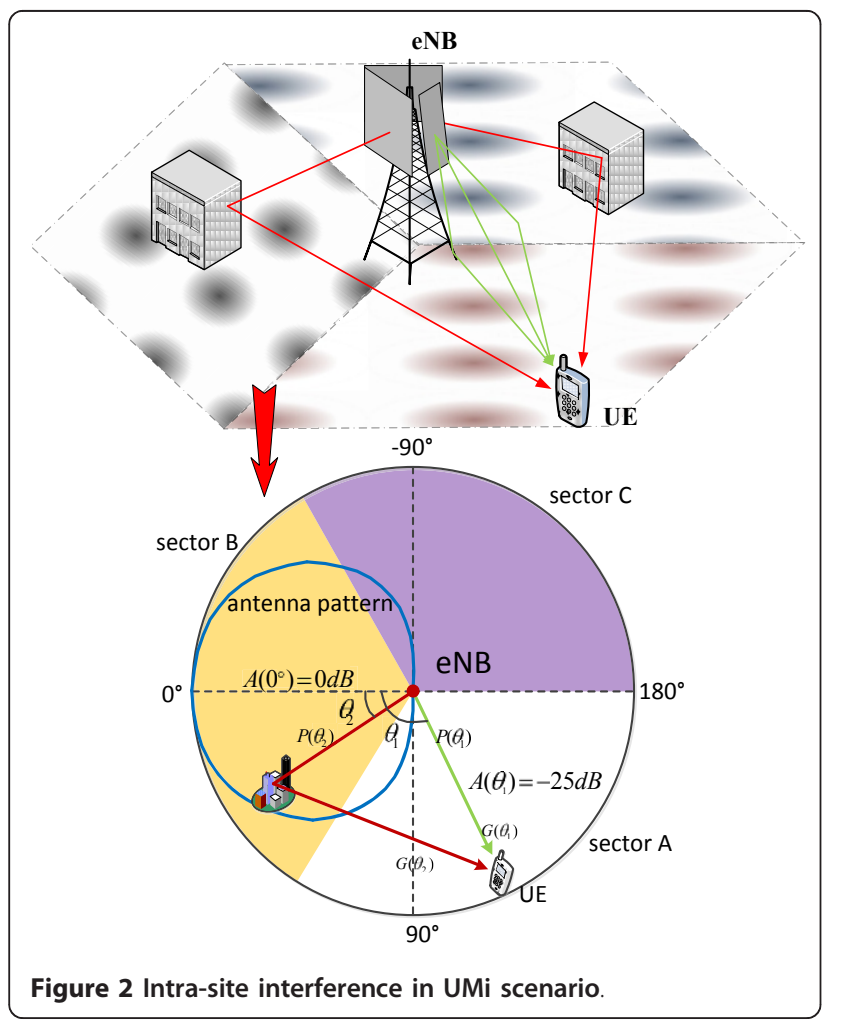

propagation channel, e.g., the center of the PAS has some offset from the LoS direction. Considering the impact of the sectored antenna pattern, it may influence the interference modeling much and thus influence the network capacity.

To facilitate the analysis, the intra-site interference from sector B is defined as follows

$$
I=X \cdot \int_{-180^{\circ}<\theta \leq 180^{\circ}} \mathrm{PL} \cdot A_{\mathrm{eNB}}(\theta) \cdot P(\theta) \cdot A_{\mathrm{UE}} \cdot d \theta,
$$

where $X$ is the transmission power of the eNB. PL is the path loss determined by the distance between eNB and UE. $A_{e N B}(\theta)$ is the antenna gain of the sector $\mathrm{B}$ at AoD $\theta \cdot P(\theta)$ is the channel gain at $\operatorname{AoD} \theta$. $A_{\mathrm{UE}}$ is the omni-directional antenna gain at UE side which can be assumed to be constant. Regarding a certain UE location, the interference can be rewritten as

$$
I=X \cdot \mathrm{PL} \cdot A_{\mathrm{UE}} \cdot \int_{-180^{\circ}<\theta \leq 180^{\circ}} A_{\mathrm{eNB}}(\theta) \cdot P(\theta) \cdot d \theta .
$$

Let $G(\theta)=A_{\mathrm{eNB}}(\theta) \cdot P(\theta)$, which is the channel gain affected by the eNB transmission antenna. Therefore, Equation 2 can be transformed into

$$
I=X \cdot \mathrm{PL} \cdot A_{\mathrm{UE}} \cdot \int_{-180^{\circ}<\theta \leq 180^{\circ}} G(\theta) \cdot d \theta .
$$

The eNB antenna pattern is usually defined as follows [9]:

$$
A(\theta)=10^{-\min \left[12\left(\frac{\theta}{\theta_{3 d B}}\right)^{2}, A_{m}\right] / 10,}
$$

where $\theta_{3} \mathrm{~dB}$ is the mainlobe's $3 \mathrm{~dB}$ beam width, and $A_{m}$ is the maximum attenuation. Typically, $\theta_{3 \mathrm{~dB}}=70^{\circ}$ and $A_{m}=25 \mathrm{~dB}$.

As illustrated in Figure 2, the green line denotes the interference departing at $\theta_{1}$ from the LoS direction, whereas the red line represents the interference departing at $\theta_{2}$. And the channel gain affected by the eNB transmission antenna at $\theta_{1}$ and $\theta_{2}$ can be calculated as $G\left(\theta_{1}\right)=A_{\mathrm{eNB}}\left(\theta_{1}\right) \cdot P\left(\theta_{1}\right)$ and $G\left(\theta_{2}\right)=A_{\mathrm{eNB}}\left(\theta_{2}\right) \cdot P\left(\theta_{2}\right)$, respectively. In conventional channel model, $P\left(\theta_{1}\right) \leq P$ $\left(\theta_{2}\right)$, thus the values of $P\left(\theta_{1}\right)$ and $P\left(\theta_{2}\right)$ determine the relative magnitude between $G\left(\theta_{1}\right)$ and $G\left(\theta_{2}\right)$ if $A_{\mathrm{eNB}}\left(\theta_{1}\right)$ and $A_{\mathrm{eNB}}\left(\theta_{2}\right)$ are not taken into account. However, according to the antenna pattern gain (the blue curve in Figure 2) depending on the departure angle of each path, it is found that $A_{\mathrm{eNB}}\left(\theta_{2}\right)$ is much larger than $A_{\mathrm{eNB}}$ $\left(\theta_{1}\right)$. So it is very possible that $G\left(\theta_{2}\right)$ could be in the same order of magnitude with $G\left(\theta_{1}\right)$. If $G\left(\theta_{2}\right)$ is ignored in the interference calculation from the channel 
modeling, the interference experienced by the UE will be inaccurate.

\section{Field MIMO channel measurement}

In order to verify our hypothesis, the dedicated field MIMO channel measurements are carried out in downtown Beijing for typical UMi scenario. This section introduces the details of the environment, equipment, and procedures of the field channel measurement.

\subsection{Measurement equipment}

The measurement was performed with the Elektrobit PropSound channel sounder, which is described in more details in [12,13]. The center frequency is $2.35 \mathrm{GHz}$, which has been allocated to the IMT-Advanced systems. Both eNB and UE employ the three-dimensional (3D) dual-polarized omni-directional arrays (ODA) with maximum 56 elements as shown in Figure 3. The element spacing of the ODA is half of a wavelength. In our measurements, eNB and UE use 16 and 32 elements of ODA, respectively, to accurately extract the 3D spatial characteristics of the MIMO channel. The antenna pattern of the ODA is calibrated in the anechoic chamber. Thus, the influence of the antenna can be excluded in data processing and the antenna-independent channel model can be constructed.

The channel sounding equipment works in a timedivision multiplexing mode. One channel sample of the whole MIMO matrix is called a cycle. Each antenna pairs is sounded once in a cycle due to that the highspeed antenna switching units at both sides, which enable the channel sounder to cap-ture the channel response of each antenna pairs during the coherent time. To capture the delay characteristics, wideband periodic pseudo-random signals are transmitted between different antenna pairs in sequence. The code length is set to 255 , which is long enough to capture all the

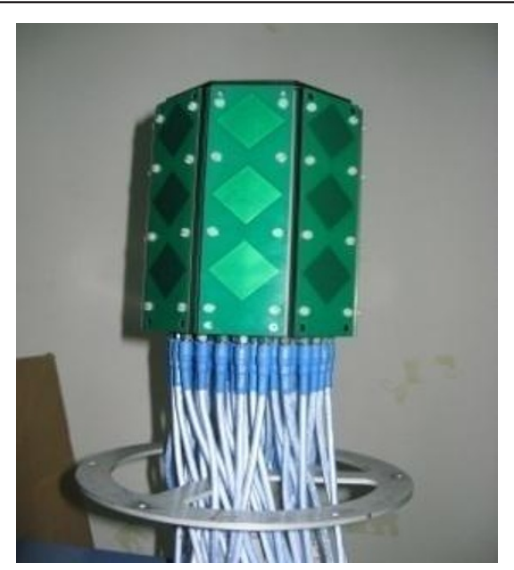

Figure 3 ODA used in measurement. propagation paths in UMi scenario. The transmitter $(\mathrm{Tx})$ and receiver $(\mathrm{Rx})$ are synchronized by an internal rubidium clock before the measurement. At Rx side, the raw data including channel information are collected. The parameter settings of the channel sounder are summarized in Table 1.

\subsubsection{Environment and measurement procedures}

To capture the propagation characteristics of the typical UMi scenario, the measurement site and scenarios are selected in downtown area of Beijing, China. The bird's eye view of the measurement environment is illustrated in Figure 4. For this scenario, the eNB is usually deployed near the corner of the cross-streets in order to provide a thorough coverage to the two roads. In our measurement, Tx is placed at the site of a GSM base station, the $\mathrm{Rx}$ is moving along the streets around the Tx as illustrated in Figure 4. To simulate a user device, the $\mathrm{Rx}$ antenna array was fixed on a trolley and moved along the routes marked with yellow color in Figure 4. The Routes 1 and 2 are under LoS condition, and Route 3 is under non-LoS (NLoS) condition. ODA is applied at both the $\mathrm{Tx}$ and $\mathrm{Rx}$ sides to capture the back side paths. The positions of Rx were recorded by global positioning system (Figure 5).

The measured data are stored in the memory of the $\mathrm{Rx}$ and the channel characteristics are extracted by accurate data post processing. LoS and NLoS cases are processed separately to analyze the possible differences.

\section{Data processing and proposed angle offset model}

In this section, the post-data processing for the field channel measurement results are introduced. The random angle offset from the LoS direction is observed from the extracted spatial parameters cycle-by-cycle. Based on the statistics of the angle offset values from an amount of measurement cycles, the empirical model is regressed and proposed to capture such spatial characteristic into the GBSM channel model.

Table 1 Measurement parameters

\begin{tabular}{ll}
\hline Items & Settings \\
\hline Carrier frequency $(\mathrm{GHz})$ & 2.35 \\
Bandwidth $(\mathrm{MHz})$ & 50 \\
Code length (chips) & 255 \\
Transmitting power $(\mathrm{dBm})$ & 26 \\
Types of antennas & ODA \\
Number of eNB antenna $\left(\mathrm{N}_{\mathrm{BS}}\right)$ & 16 \\
Number of MS antenna $\left(\mathrm{N}_{\mathrm{MS}}\right)$ & 32 \\
Height of eNB antenna $(\mathrm{m})$ & 7 \\
Height of MS antenna $(\mathrm{m})$ & 1.8 \\
\hline
\end{tabular}




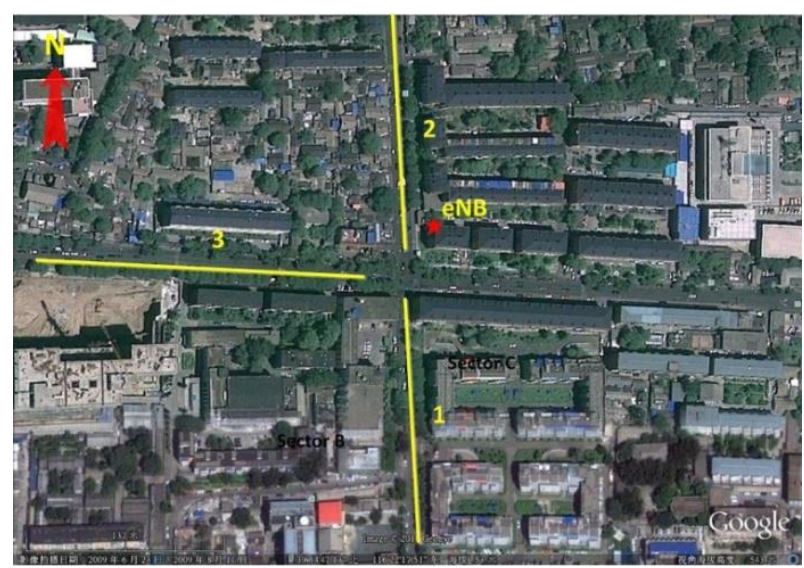

Figure 4 Bird's eye view of the measurement scenario in downtown Beijing. (Photo courtesy of Google Earth.)

\subsection{Data processing}

In data post processing, firstly, the CIRs are converted from the raw data by sliding correlating the received signals with a synchronized copy of the sequence. Then Space-Alternating Generalized Expectation maximization (SAGE) algorithm [14-17], which has widely been used for channel parameter estimation, is applied in order to extract the channel parameters from the CIRs. As an extension of Maximum-Likelihood (ML) method, the SAGE algorithm provides a joint estimation of the parameter set with no constrains on the response of antenna array. $\tau_{n}, v_{n}, \varphi_{n}, \phi_{n}, \mathbf{A}_{n}, P_{n}$ denote the propagation delay, Doppler shift, AoD, AoA, polarization matrix, and the power of the $n$th prop-agation path, respectively.

From the extracted parameters, we can acquire the channel power at AoD $\theta$ as

$$
P(\theta)=\frac{\sum_{n=1}^{N(\theta)} P_{n, \theta}}{\sum_{n, \theta} P_{n, \theta}}
$$

where $N(\theta)$ is the number of paths of which the $\varphi_{n}=\theta$, and the $P_{n, \theta}$ is the power of the corresponding path.

\subsection{Data analysis and angular offset modeling}

Figure 6 depicts a spatial sample of the SAGE results. The points with dif-ferent colors stand for distinct paths extracted in one cycle in polar coordinate system, with total of 50 paths. The angles of those points are the $\varphi_{n}$ from SAGE results. Besides, the radius of each point means the power of the path, $P_{n}$. It is clearly shown that there are two dominant groups of paths in this cycle, where one group, drawn with black heavy line, coincides with the LoS direction, and the other group reaches the UE through reflection of buildings in the street.
It is reasonable to assume that the mean angle of all existent paths locates approximately in the middle of the two groups, which means that the center of the PAS distribution in this environment is not in accordance with the LoS direction. It can be explained by the fact that in the crossroads environment, most of the propagation paths come along the street and are at the same side of the LoS line. Thus, the offset between the mean angle of PAS distribution and LoS direction appears and it determines the amount of signals deflected to nearby sectors. In typical UMi environment, since the eNB is deployed at the roof top, where lots of buildings are higher than the eNB, offset values can easily be observed for the canyon propagation in the street.

However, in the existing channel models such as IMTAdvanced channel model, it assumes that the PAS follows wrapped Gaussian or Laplacion distribution with its center along the LoS direction. The offset between the mean angle of PAS distribution and LoS direction is ignored.

In order to quantitatively describe this offset, the angular spread of departure (ASD) is defined and calculated with the equation [9]:

$$
\phi_{\mathrm{rms}}=\min _{\Delta \phi}\left(\sqrt{\frac{\sum_{n}\left(r\left(\phi_{n}+\Delta \phi-\phi_{\text {mean }}\right)\right)^{2} P_{n}}{\sum_{n} P_{n}}}\right)
$$

where $\varphi_{n}$ is the AoD, the minimization over $\Delta \varphi$ is to eliminate the additional angle spread introduced by different selection of reference zero angle. $r(\cdot)$ convert angles to $(-180,180)$ degree. $\varphi_{\text {mean }}$ is the power weighted mean angle which is derived from $\phi_{\text {mean }}=\sum_{n}^{N} r\left(\phi_{n}+\Delta \phi\right) P_{n} / \sum_{n}^{N} P_{n}$.

For the PAS that follows wrapped Gaussian or Laplacian distribution, $\varphi_{\text {rms }}$ will reach its minimum value 


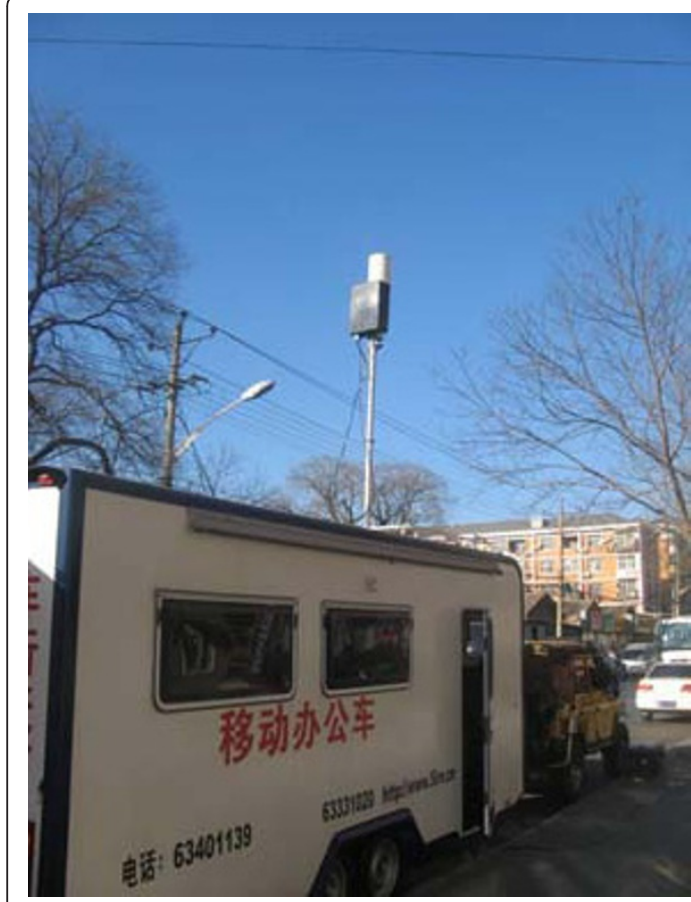

(a) $\mathrm{Tx}$

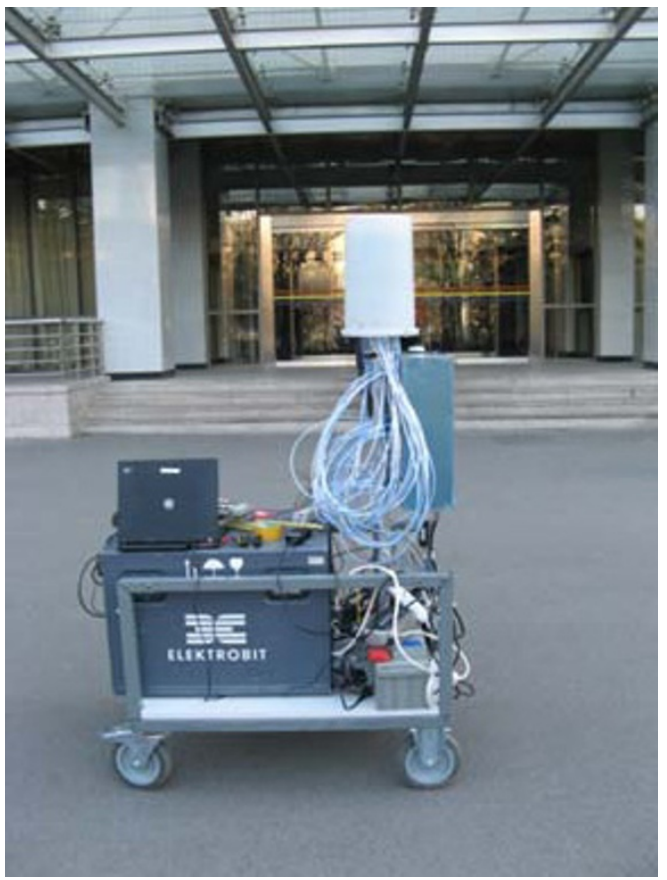

(b) $R x$

Figure 5 Pictures of measurement environment. (a) $T x_{;}$(b) $R x$

when $\Delta \varphi$ is at the center of the distribution. Thus, during the data processing of the measurement data, we can obtain the $\Delta \varphi$ that minimizes $\varphi_{\mathrm{rms}}$, which is also the center angle of the PAS distribution.

To find the mean angle denoted in Figure 6, i.e., the center of all paths in one cycle, the coordinate system in which the angle spread is minimized need to be

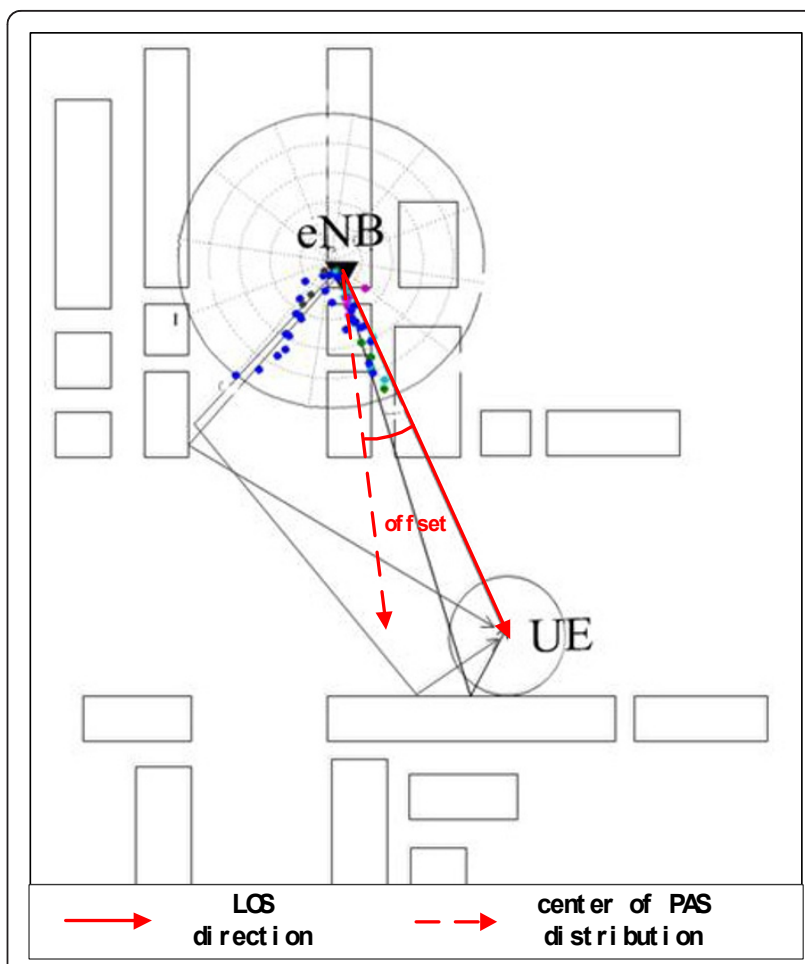

Figure 6 Illustration of angular offset under realistic environment.

determined. Offset values are obtained by subtracting the mean angles of all paths from LoS direction in the coordinate system.

According to the measurement result, it is found that the distribution center is not in accordance with the LoS direction (referred as 0 degree) and an angular offset is observed. In current channel models, the ASA and ASD are calculated with Equation 6 from measured data, and when we use the channel model to generate $\varphi_{n}$, the center of the Gaussian or Laplacian distribution is assumed to be the LoS direction, which is in contradiction with our observation.

In Figure 7, the examples of measured offset angle for one LoS route and one NLoS route are shown. It can be seen that for LoS route the offset angle is quite near 0 degree, but for NLoS routes the offset angle is highly depend on the specific environment and usually has a large value. It means that for LoS scenarios, maybe the offset angle can be neglected just as the previous modeling method does. However, for NLoS cases, it is unreasonable to neglect the offset angle with such a large value. Especially for the UMi scenario at the crossroads, where the angle offset is very common and has its physical explanation that almost all the propagation paths are arriving along the streets.

To capture such spatial characteristic into the MIMO channel model, the offset values in each cycle are collected 


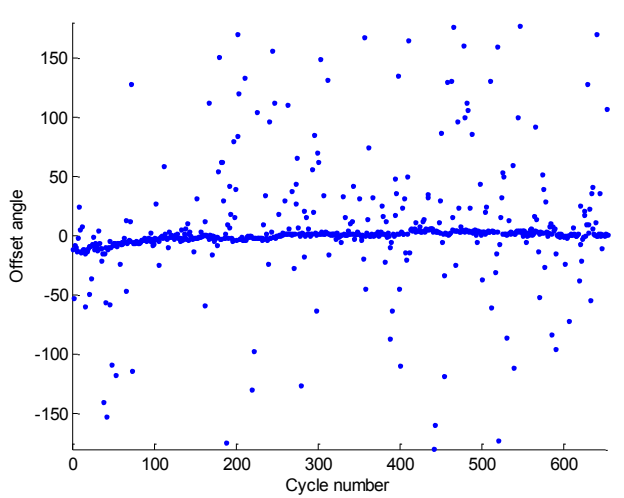

(a) LoS

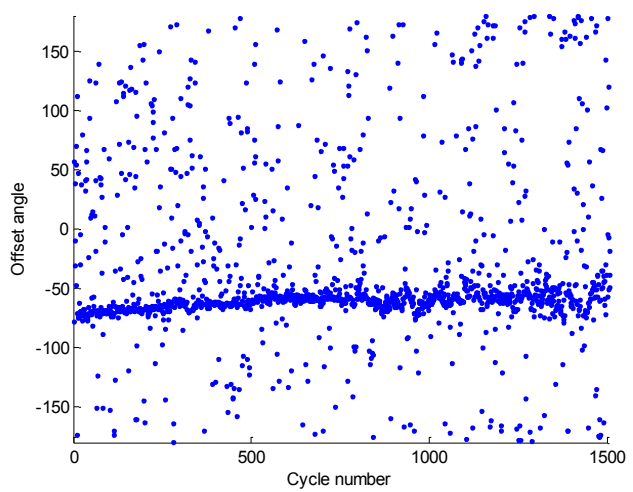

(b) NLOS

Figure 7 Angle offset for LoS and NLoS routes. (a) LOS; (b) NLOS.

and its empirical distribution is regressed out for modeling. Probability density function of the offset values and their best fits under LoS and NLoS cases are shown in Figures 8 and 9. Due to the symmetry of the network architecture, only absolute offset values are considered here.

For LoS case, the absolute value of angular offset $\alpha$ can be regressed as log-logistic distribution, i.e.,

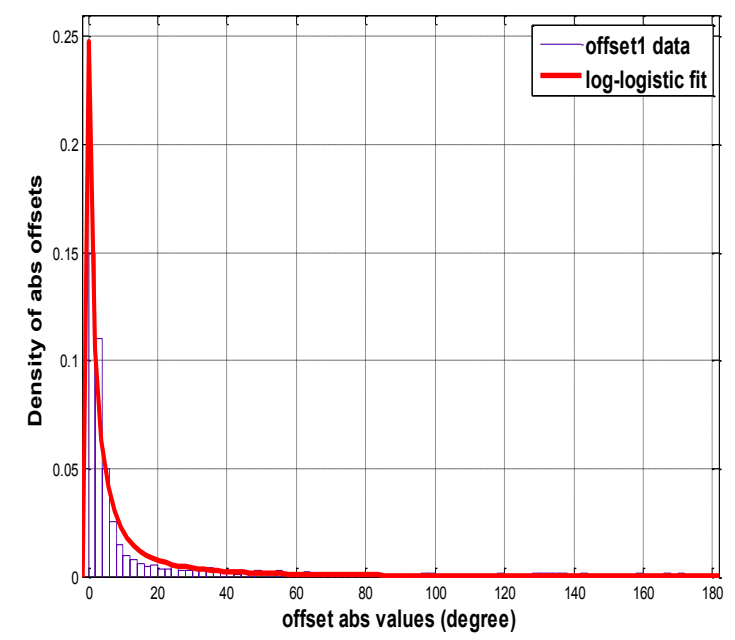

Figure 8 Absolute offset values under LoS environment. $f(\alpha)=\frac{e^{\frac{\ln \alpha-\mu}{\sigma}}}{\alpha \sigma\left(1+e^{\frac{\ln \alpha-\mu}{\sigma}}\right)^{2}}$

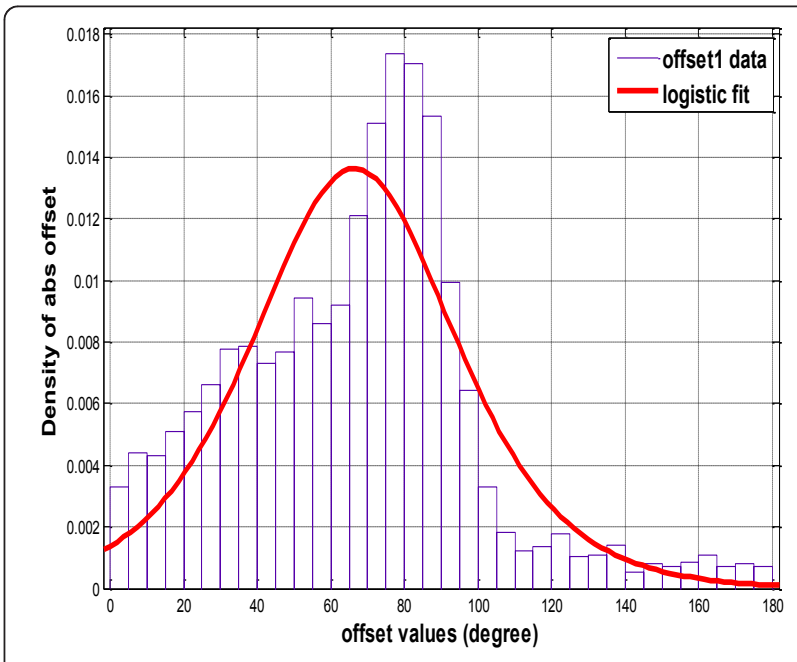

Figure 9 Absolute offset values under NLoS environment. 
where $\mu=1.524$ and $\sigma=1.048$ are extracted from the measured results. The $\alpha$ can be set as positive and negative values in equal probability.

For NLoS case, the absolute value of $\alpha$ can be regressed as logistic distribu-tion:

$$
f(\alpha)=\frac{e^{\frac{\alpha-\mu}{\sigma}}}{\sigma\left(1+e^{\frac{\alpha-\mu}{\sigma}}\right)^{2}}
$$

where $\mu=66.461$ and $\sigma=18.325$. The $\alpha$ can be set as positive and negative values in equal probability.

It is shown that under LoS environment, the absolute values of the offset angles are best fitted with log-logistic distribution, while there are best fitted with logistic distribution under NLoS environment. So, such spatial characteristic of typical UMi scenario can be captured into the IMT-Advanced channel model with the proposed distribution.

In current ITU-R IMT-Advanced channel model, the downlink AoD of the $m$ th subpath of the $n$th path, $\varphi_{n}$, $m$, is generated LoS-centered. To include the offset, we can simply replace all the $\varphi_{n, m}$ generated in one drop with

$$
\phi_{n, m}^{\prime}=\phi_{n, m}+Y \cdot \alpha
$$

where $\alpha$ is randomly generated according to the distributions described by Equation 9 for LoS or Equation 10 for NLoS. A random variable $Y$ with uniform distribution in the discrete set of $\{1,-1\}$ is multiplied to assign positive or negative sign to the offset angles. Since the offset is an overall shift of the PAS in one drop, it should be noted that the offset angle $\alpha$ is generated for each link and all the $\varphi_{n, m}$ in the same link should be shifted with the same offset angle (all the AoD of the paths have the same offset). The generation of all the other parameters can be the same as the original IMT-Advanced channel model.

The proposed angular offset models and parameters for UMi scenario are summarized in Table 2.

To verify the necessity of this offset modeling to the channel modeling and the corresponding network performance modeling, theoretical capacity and network capacity analysis are conducted; the results are presented in the following section.

Table 2 Proposed parameters

\begin{tabular}{llll}
\hline Items & LoS & NLoS \\
\hline \multicolumn{2}{l}{ Angular offset distribution } & Log-logistic & Logistic \\
Parameters $\left(^{\circ}\right)$ & $\mu$ & 1.524 & 66.461 \\
& $\sigma$ & 1.048 & 18.325 \\
\hline
\end{tabular}

\section{Impact of angular offset on channel capacity}

To evaluate the impact of angular offset under different kinds of antenna configurations and different antenna patterns, the 50 paths extracted from SAGE algorithm, where the impact of the measurement antenna is excluded, are used to reconstruct the channel of desired antenna setup.

It should be noted that before applying the sectored antenna pattern, zero angle direction should be adjusted as the bisection of the specified sector. Referring to [9], the channel reconstruction is given as follows:

$$
\begin{aligned}
\mathbf{H}(\tau)_{u, s} & =\sum_{n=1}^{N(\tau)} \mathbf{F}_{R x, u}^{T}\left(\varphi_{n}\right) \mathbf{A}_{n} \mathbf{F}_{T x, s}\left(\phi_{n}\right) \\
& \cdot \exp \left(j d_{s} 2 \pi \lambda_{0}^{-1} \sin \left(\phi_{n}\right)\right) \\
& \cdot \exp \left(j d_{u} 2 \pi \lambda_{0}^{-1} \sin \left(\varphi_{n}\right)\right)
\end{aligned}
$$

where $\tau_{n}, \varphi_{n}, \phi_{n}$ are extracted by SAGE algorithm. Moreover, $(\cdot)^{T}$ denotes the matrix transpose; $N(\tau)$ represents the number of paths at the given delay $\tau$; $\lambda_{0}$ is the wavelength of the carrier, and $d_{u}$ stands for the distance between the $u$ th $\mathrm{Rx}$ antenna element and the first element; $\mathbf{F}_{R x, u}$ is the field pattern of the $\mathrm{Rx}$ antenna element. For Tx antenna elements, $d_{s}$ and $\mathbf{F}_{T x}$, s hold the same meanings with $d_{u}$ and $\mathbf{F}_{R x, u}$, respectively. In the following studies, all $\mathrm{Tx}$ and $\mathrm{Rx}$ antennas are assumed to be isotropic for simplicity, i.e., the field patterns can be rewritten as [9]

$$
\begin{aligned}
& \mathbf{F}_{T x, s}\left(\phi_{n}\right)=\left[\begin{array}{l}
\mathbf{F}_{T x, s, v}\left(\phi_{n}\right) \\
\mathbf{F}_{T x, s, h}\left(\phi_{n}\right)
\end{array}\right]=\left[\begin{array}{c}
\cos \beta_{T x} \\
\sin \beta_{T x} \cos \phi_{n}
\end{array}\right] \\
& \mathbf{F}_{R x, u}\left(\varphi_{n}\right)=\left[\begin{array}{l}
\mathbf{F}_{R x, u, v}\left(\varphi_{n}\right) \\
\mathbf{F}_{R x, u, h}\left(\varphi_{n}\right)
\end{array}\right]=\left[\begin{array}{c}
\cos \beta_{R x} \\
\sin \beta_{R x} \cos \varphi_{n}
\end{array}\right]
\end{aligned}
$$

where $\beta$ indicates the slant angle between the antenna element and the vertical direction. Finally, $\mathbf{H}(\tau)$ is converted into frequency domain by applying Discrete Fourier Transform, i.e., $\mathbf{H}_{\text {recon }}(f)$.

$\varphi_{n}$ denotes the angle difference between the $n$th path and LoS direction at the transmitter, respectively. If the offset values are not taken into consideration, just as the traditional channel models do, the coordination system is shifted to make the center of the paths coincide with LoS direction. $\varphi_{n}$ is then expressed as

$$
\phi_{n}^{\prime}=\phi_{n}-\alpha_{i}
$$

where $\alpha_{i}$ means the offset value of the cycle $i$. Thus, the comparison of the channel capacity with and without the offset angle can be considered as the comparison of the proposed model and the traditional model.

For capacity analysis, we assume that there is no channel state information (CSI) available at the transmitter 
side. The transmit power is uniformly allocated across frequency and space. Thus, with the reconstructed channel matrix, the capacity of the link can be expressed as [18]

$$
C=\frac{1}{B} \int_{B} \log _{2} \operatorname{det}\left[I_{U}+\frac{\rho}{U} \mathbf{H}_{\text {recon }}(f) \mathbf{H}_{\text {recon }}^{*}(f)\right] \mathbf{d} f
$$

where $\mathbf{H}_{\text {recon }}(f)$ is acquired by transforming the reconstructed channel impulse response into frequency domain.

Besides, from the eigenvalues of the channel matrix, we can get a better understanding of the eigen multipath channels which determines the performance of beamforming. Therefore, the cumulative distributive function (CDF) of the ratio of the biggest eigenvalue and the smallest eigenvalues is also presented.

To verify the angular offset on the theoretical channel capacity, the MIMO channel statistics for two cases are compared. Case 1 is named as "measured data excluding offset", which is equivalent to the original IMTAdvanced UMi channel model, where the angle offset from the LoS direction is ignored. The case 2 is named as "measured data", which is equivalent to the modified IMT-Advanced UMI channel model, where the offset value from the LoS direction is taken into account for the fast fading modeling.

As shown in Figure 10, CDF of capacity for these two cases is given. It can be observed that the impact of angular offset value is negligible when SNR is $-5 \mathrm{~dB}$ since the system suffered from heavy noise. When the SNR improves, the capacity calculated directly from the measured data is larger than that when angular offset is excluded. In other words, the current channel models might underestimate the channel capacity by ignoring the angular offset.
In Figure 11, the ratios of the two eigenvalues of the channel matrix are presented. It shows that when angular offset is excluded, the ratio of the eigenvalues turns to be larger which implies that the power of the channel is more centralized on one of the two eigen spaces. It is in line with the results in Figure 10, when power is equally allocated in each antenna element, smaller ratio of the eigen values will lead to larger capacity.

\section{Impact of angular offset on network capacity}

To verify the impact of angular offset on the network interference and capacity, the dedicated network simulation is conducted. The details of the simulation can be found in [19].

Figure 12 is the typical cellular deployment scenario used in 3GPP. The interference from the other two sectors within the same site is defined as intra-cell interference, while the interference from the other sites is defined as inter-cell interference. The ratio of the intra-site interference to inter-site interference is defined as

$$
R=\frac{I_{\text {intra }}}{I_{\text {inter }}}
$$

From the simulation results illustrated in Figure 13, the proposed angular offset has much impact on the proportion of intra-site interference. The IMT-Advanced UMi channel model without considering the angular offset leads to underestimation on the intra-site interference. For an intra-site CoMP system, where the three sectors belonging to the same eNB can coordinate to transmit and receive simultaneously, the intra-site interference can be transformed into useful signals in intrasite CoMP system. Therefore, the underestimation on the intra-site interference will lead to underestimation

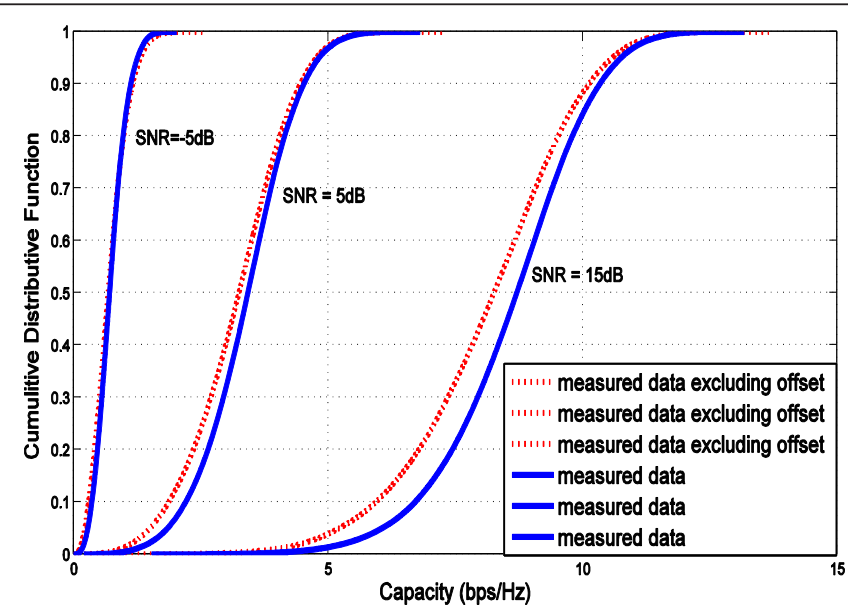

Figure 10 Comparison of CDF for capacity with and without angular offset. 


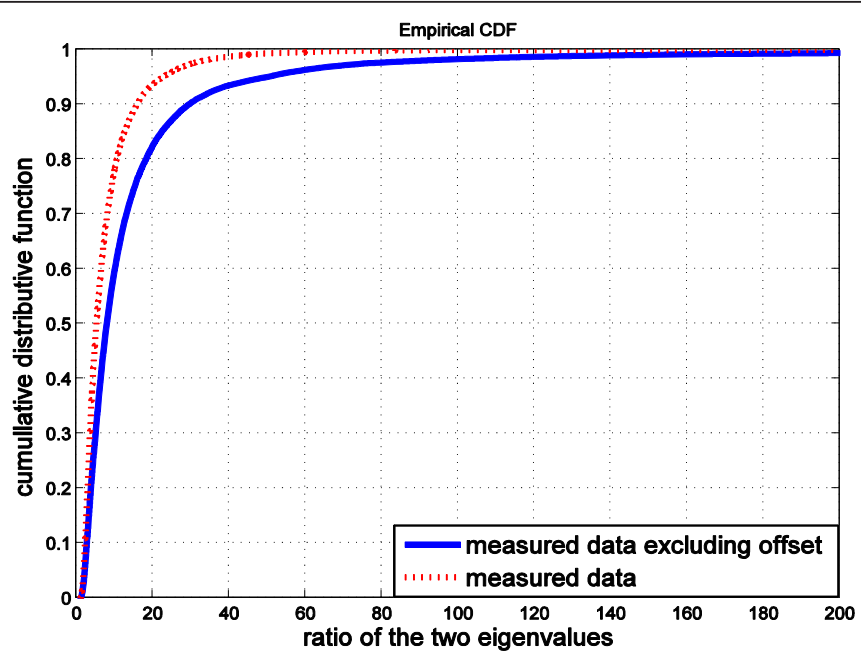

Figure 11 Comparison of ratio of the two eigen values of the channel matrix.

on the intra-site CoMP performance gain over the conventional single point MIMO system.

To verify the impact of the angular offset on the system performance, homogeneous intra-site CoMP in UMi scenario is simulated. To facilitate the comparison, TD-LTE-Advanced system [11] is assumed for the simulation. The antenna configuration is $4 \times 2$ in downlink. For intra-site CoMP, the joint processing with block diagonalization algorithm is adopted [19] since the downlink CSI can be obtained at the eNB by uplink sounding. The detailed evaluation methodology and configurations of UMi can be found in [9]. For TD LTE-Advanced, the sounding period is $5 \mathrm{~ms}$, dual-polarized antennas are adopted at both eNB and UE, and the antenna spacing is half a wavelength. The main assumptions for the simulation are listed in Table 3.

To show the impact of the proposed angular offset model, the simulations based on the existing IMTAdvanced UMi channel models and the modified IMTAdvanced UMi channel models are performed independently.

From the simulation results in Table 4, it is found that the cell average spectrum efficiency of the single point MIMO system has been overestimated by $20 \%$ and the cell edge spectrum efficiency is overestimated by $71 \%$. The cell average spectrum efficiency of intra-site CoMP has similar performance, whereas the cell edge spectrum efficiency is underestimated by $61 \%$ (Table 3 ).

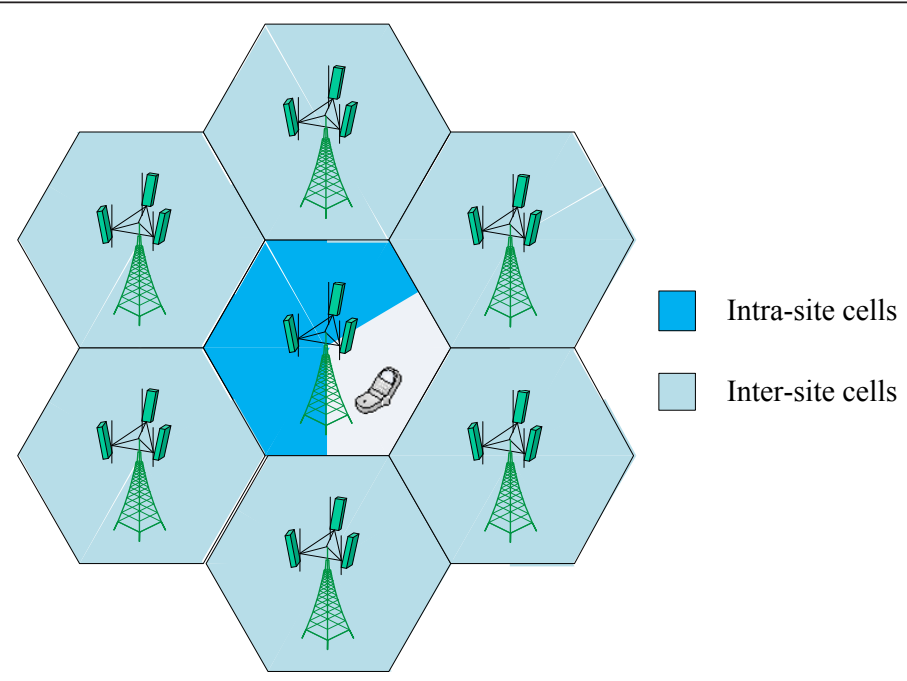

Figure 12 Demonstration on intra-site and inter-site interference. 


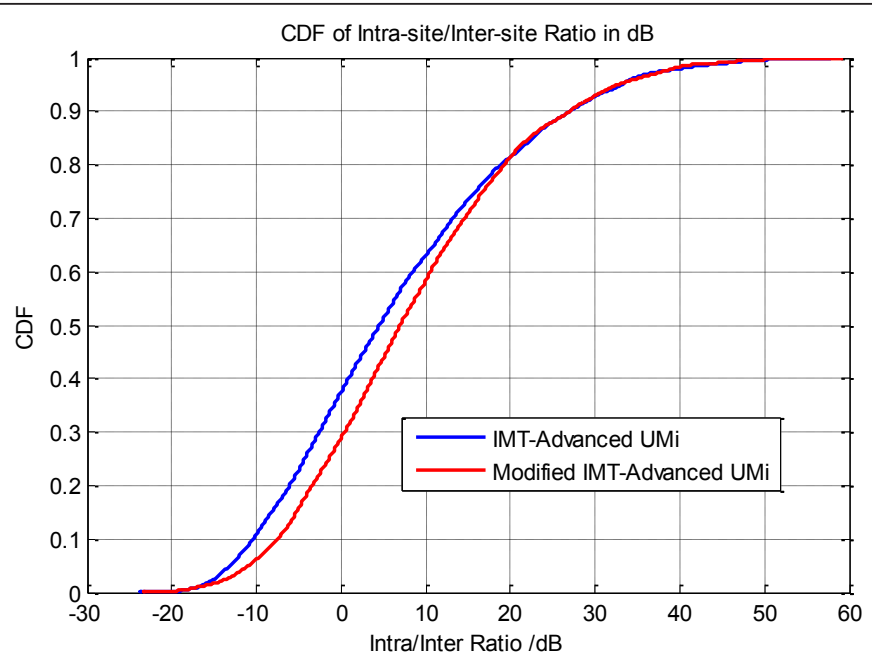

Figure 13 Impact of angular offset on ratio of intra-site interference to inter-site interference.

From Table 3, it is also found that the performance gain of MU-CoMP over EBB is underestimated by 55 and $80 \%$, respectively, in spectrum efficiency and cell edge spectrum efficiency by conventional IMTAdvanced UMi channel model. Whereas 44 and $87 \%$ performance gain of CoMP over MU-BF has been underestimated by IMT-Advanced UMi channel model.

The conclusion based on the analysis and simulation results above quite align with our original hypothesis. The current GBSM without considering the angular offset underestimates the intra-site interference of the UMi scenario due to the special characteristics of the environment and the impact of the directional antenna pattern. The proposed angular offset is necessary for the IMT-Advanced UMi model to capture the spatial characteristic of the scenario better and improve the accuracy of the channel modeling for UMi of Hetnet.

\section{Conclusions}

For HetNet research and performance evaluation, accurate channel model is very important. However, the current standardization work only explicitly specifies the path loss and shadowing model. To evaluate the solutions in spatial and frequency domain and to investigate the interference effects in HetNet scenarios, the accurate fast fading model is vital. This literature addresses the spatial propagation modeling for UMi of HetNet. Based on the analysis on angular offset characteristics of the UMi scenario and the impact of eNB transmitter antenna pattern on the interference modeling, it is concluded that the existing GBSM model, e.g., IMTAdvanced UMi channel model, leads to underestimated

Table 3 Simulation parameters

\begin{tabular}{ll}
\hline Parameter & ITU-R UMi \\
\hline Site layout & 3 -sectorized hexagonal grid with 7 cells and wrap-around \\
Operating bandwidth & $10 \mathrm{MHz}$ \\
DL/UL ratio & $2 \mathrm{DL} / 2 \mathrm{UL}$ \\
Special subframe & {$[10: 2: 2]$ for DwPTS, GP and UpPTS } \\
UpPTS & \\
Antenna boresight points toward flat side of cell & \\
Antenna pattern & Polarized antenna/horizontal antenna \\
eNB transmission power & $46 \mathrm{dBm}$ \\
UE receiver structure & Minimum mean square error \\
UE number & $10 /$ sector in full queue \\
Link-to-system interface for simulations & Ml-ESM \\
HARQ combining & Chase combining \\
Penetration loss & $20 \mathrm{~dB}$ \\
\hline
\end{tabular}

Details can be found in [19]. 
Table 4 Impact of the proposed angular offset model

\begin{tabular}{llllllll}
\hline Simulation case & \multicolumn{1}{l}{$\begin{array}{l}\text { IMT- } \\
\text { Advanced } \\
\text { UMi }\end{array}$} & \multicolumn{2}{l}{$\begin{array}{l}\text { Proposed } \\
\text { UMi }\end{array}$} & \multicolumn{2}{l}{$\begin{array}{l}\text { Performance } \\
\text { Loss }\end{array}$} \\
\cline { 2 - 8 } & & SE & CSE & SE & CSE & SE & CSE \\
\hline UMi & EBB(bps/Hz) & 1.81 & 0.054 & 1.45 & 0.016 & $-20 \%$ & $-71 \%$ \\
& MU-BF(bps/Hz) & 2.91 & 0.088 & 2.21 & 0.022 & $-24 \%$ & $-75 \%$ \\
& MU-CoMP(bps/Hz) & 4.38 & 0.135 & 4.31 & 0.053 & $-2 \%$ & $-61 \%$ \\
\hline
\end{tabular}

Note: $\mathrm{SE}$, spectrum efficiency; CSE, cell edge spectrum efficiency; EBB, eigen value-based beamforming; MU-BF, multiuser beamforming; MU-CoMP, multiuser CoMP.

intra-site interference. To verify this, field MIMO channel measurements are carried out in downtown Beijing for typical UMi scenario. From the measurements, the angular offset of the multipath is clearly observed. In the proposed model, the angular offset in LoS and NLoS cases are modeled as log-logistic and logistic distribution, respectively. The theoretical channel and the network capacities' analysis proves that it is necessary to capture the random angle offset modeling into the IMTAdvanced UMi channel model.

\section{Acknowledgements}

This study was supported in part by the China Important National Science and Technology Specific Projects under Grant No. 2009ZX03007-003-01 and by China 863 Program and Major Project under Grant No. 2009AA011502.

\section{Author details}

'Key Laboratory of Universal Wireless Communications, Ministry of Education, Beijing University of Posts and Telecommunications, P.O. Box 92, Beijing 100876, China ${ }^{2}$ China Mobile Communications Corporation, Beijing, China ${ }^{3}$ Orange-France Telecom, San Francisco, CA, USA

\section{Competing interests}

The authors declare that they have no competing interests.

Received: 2 March 2011 Accepted: 28 November 2011

Published: 28 November 2011

\section{References}

1. M Iwamura, K Etemad, F Mo-Han, R Nory, R Love, Carrier aggregation framework in 3GPP LTE-advanced. IEEE Commun Mag. 48(8), 60-67 (2010)

2. Z Zhao, J Wang, H Guan, P E Mogensen, G Liu, X Shen, TD-LTE Network Deployment Evolution in a Metropolitan Scenario. in Proceedings of the IEEE Vehicular Technology Conference (VTC-Fall) USA 1-5 (2011)

3. 3GPP TR 36.806, Evolved universal terrestrial radio access (E-UTRA); relay architectures for E-UTRA (LTE-Advanced) (2010)

4. R Pabst, BH Walke, DC Schultz, P Herhold, H Yanikomeroglu, S Mukherjee, H Viswanathan, M Lott, W Zirwas, M Dohler, H Aghvami, DD Falconer, GP Fettweis, Relay-based deployment concepts for wireless and mobile broadband radio. IEEE Commun Mag. 42(9), 80-89 (2004). doi:10.1109/ MCOM.2004.1336724

5. ITU-R Report M.2133, Requirements, evaluation criteria and submission templates for the development of IMT-Advanced (2008)

6. 3GPP TR 36.814, Evolved universal terrestrial radio access (E-UTRA): further advancements for E-UTRA physical layer aspects (2010)

7. S Venkatesan, Coordinating base stations for greater uplink spectra efficiency in a cellular network. in Proc IEEE 18th International Symposium Personal, Indoor and Mobile Radio Communications PIMRC 2007 1-5 (2007)

8. 3GPP RAN1 R1-111086, CoMP Simulation assumptions, NTT DoCoMo (2011)
9. ITU-R report M.2135, Guidelines for evaluation of radio interface technologies for IMT-Advanced (2009)

10. ITU-R report M.1225, Guidelines for evaluation of radio transmission technologies for IMT-2000 (1997)

11. ITU-R WP5D Document 5D/580, Submission of TD-LTE-Advanced for IMTAdvanced candidate technology, China (2010)

12. A Stucki, PropSound system specifications document: concept and specifications, Switzerland, Technical Report. (2001)

13. Elektrobit, Propsound multidimensional channel sounder. http://www. propsim.com

14. BH Fleury, M Tschudin, R Heddergott, D Dahlhaus, K Ingeman Pedersen, Channel parameter estimation in mobile radio environments using the sage algorithm. IEEE J Sel Areas Commun. 17(3), 434-450 (1999). doi:10.1109/ 49.753729

15. BH Fleury, X Yin, KG Rohbrandt, P Jourdan, A Stucki, Performance of a highresolution scheme for joint estimation of delay and bidirection dispersion in the radio channel, in Proceedings of the IEEE Vehicular Technology Conference (VTC-Spring) Korea. 1, 522-526 (May 2002)

16. BH Fleury, P Jourdan, A Stucki, High-resolution channel parameter estimation for MIMO applications using the SAGE algorithm. in Proceedings of International Zurich Seminar on Broadband Communications Switzerland. 30, 1-9 (February 2002)

17. BH Fleury, X Yin, P Jourdan, A Stucki, High-resolution channel parameter estimation for communication systems equipped with antenna arrays. in Proceedings of the 13th IFAC Symposium on System Identification (SYSID), no. ISC-379, Netherlands (2003)

18. W Roh, A Paulraj, MIMO channel capacity for the distributed antenna. in Proceedings of the IEEE Vehicular Technology Conference (VTC-Fall) Canada. 2, 706-709 (September 2002)

19. Q Wang, D Jiang, G Liu, Z Yan, Coordinated multiple points transmission for LTE-Advanced systems. in Proceedings of the IEEE Int Conf Communications workshop on LTE-Advanced, Germany 1-4 (2009)

doi:10.1186/1687-1499-2011-187

Cite this article as: Zhang et al:: Analysis and modeling of spatial characteristics in urban microscenario of heterogeneous network. EURASIP Journal on Wireless Communications and Networking 2011 2011:187.

\section{Submit your manuscript to a SpringerOpen ${ }^{\circ}$ journal and benefit from:}

- Convenient online submission

- Rigorous peer review

- Immediate publication on acceptance

- Open access: articles freely available online

- High visibility within the field

- Retaining the copyright to your article

Submit your next manuscript at $\gg$ springeropen.com 